\title{
High-Temperature Magnetostriction of Magnetite and Titanomagnetites
}

\author{
BRUCE M. MOSKOWITZ
}

\author{
Department of Geology and Geophysics and Institute for Rock Magnetism, University of Minnesota at Minneapolis-St. Paul
}

\begin{abstract}
Magnetostriction of synthetic polycrystalline magnetite and titanium-rich titanomagnetites has been measured from $25^{\circ} \mathrm{C}$ to their Curie temperatures. These are the first reported measurements of magnetostriction above room temperature for titanomagnetites. The magnetostriction constants are measured with a recording rotating field magnetostrictometer, which uses a strain gage bridge technique and two rotating $\mathrm{SmCo}_{5}$ permanent magnets. Magnetostriction is recorded continuously as the sample is slowly heated and cooled in a fixed magnetic field. Measurements were made on sintered polycrystalline samples of magnetite, titanomagnetite $\left(\mathrm{Fe}_{3-\mathrm{x}} \mathrm{Ti}_{3} \mathrm{O}_{4}\right.$ $x=0.0,0.4$, and 0.6 , and an aluminum-magnesium substituted titanium-rich titanomagnetite (AMTM60). The thermal dependence of the polycrystalline magnetostriction constant $\lambda_{v}(T)$ varies as $\left(1-T / T_{c}\right)^{1.3}$ for titanium-rich titanomagnetites and as $\left(1-T / T_{c}\right)^{n g}$ for pure magnetite. The polycrystalline magnetostriction constant is well approximated by a power law equation in terms of the saturation magnetization, $\lambda_{2}(T) \propto M_{1}(T)^{2}$, where $n=2.31$ (TM0), 3.47 (TM40), 2.75 (TM60), and 2.80 (AMTM60). The magnetostriction results for AMTM60 are in excellent agreement with previous predictions based on domain observations.
\end{abstract}

\section{INTRODUCTION}

Magnetostriction is the change in crystal dimensions that accompanies the process of magnetization. It can include both linear dimensional changes and volume changes. The linear saturation magnetostriction constant $\lambda$ is the fractional change in length $\Delta / / \ell$ of a material when it is magnetized from demagnetized state to saturation. It may be positive (elongation) or negative (contraction) and, in single crystals, is usually anisotropic. Magnetostriction occurs in almost all ferromagnetic and ferrimagnetic materials below their Curie temperatures and, like magnetocrystalline anisotropy, has its origin in spin-orbit coupling [e.g., $O^{\prime}$ Reilly, 1984]. In magnetite and titanomagnetites, $\lambda$ is of the order of $10^{-5}$ at room temperature [Syono, 1965; Klapel and Shive, 1974; Klerk et al., 1977].

The effects of stress on magnetic properties are complex. However, in general, the magnitude of the effect is proportional to the product of the stress level and the magnetostriction constants. Stress may be externally applied or it may be residual. Residual stresses may also be divided as macrostresses or microstresses depending on scale [e.g., Cullity, 1972; Xu and Merrill, 1989]. Macrostresses can create a stress induced uniaxial anisotropy if the stress is reasonably uniform in direction and magnitude over distances many times the grain diameter and large enough to overcome the intrinsic magnetocrystalline anisotropy. Macrostresses may be responsible for the unusual domain style that is observed in titanium-rich titanomagnetites [Appel and Soffel, 1984; Halgedahl, 1987; Appel, 1987; Moskowitz et al., 1988]. Microstresses vary rapidly over distances less than the grain diameter. The most common source of microstress is the stress fields associated with crystalline defects, such as dislocations. Microstresses affect the movement of domain walls and therefore contribute to important macroscopic properties such as susceptibility, thermoremanence, and coercivity [Stacey and Wise, 1969; Hodych, 1982, 1986; Xu and Merrill, 1989, 1990].

Recent studies on the temperature dependence of magnetic domain structure in magnetite and titanomagnetite have shown that the thermal dependence of the exchange, magretccrystalline, ano

Copyright 1993 by the American Geophysical Union.

Paper number 92JB01846.

0148-0227/93/92JB-01846\$05.00 magnetostriction constants between room temperature and Curie temperature are fundamentally important for our understanding of remanence acquisition and its stability over geologic time [Hodych, 1982, 1986; Moskowitz and Halgedahl, 1987; Halgedahl, 1987, 1991; $X u$ and Merrill, 1989, 1990]. It is in this temperature range that the acquisition of thermoremanent magnetization occurs. Yet, experimental determinations of these constants and their thermal behavior are poorly known. Magnetostriction constants of magnetitc and titanomagnetite have been measured in several studies primarily at temperatures below room temperature [Bickford et al., 1955; Syono, 1965; Flanders, 1974; Klerk et al., 1977]. These studies have contributed to understanding the origin of magnetostriction in the titanomagnetite solid solution series but have not addressed the equally important problem of the thermal dependence of $\lambda$ above room temperature. In contrast, Klapel and Shive [1974] reported high-temperature magnetostriction measurements on pure magnetite single crystals. They observed that $\lambda_{111}$ decreased almost linearly with temperature up to the maximum temperature of $500^{\circ} \mathrm{C}$. However, the results for $\lambda_{100}$ were inconclusive, and hence $\lambda_{2}(T)$ could not be determined from these measurements. No measurements of magnetostriction have yet been reported for titanomagnetites above room temperature and approaching the Curie temperature.

In this paper, high-temperature magnetostriction measurements are reported for synthetic polycrystalline samples of pure magnetite and several titanomagnetites. The temperature range of the measurements is from room temperature to the Curie temperature. The magnetostriction constants are measured with a recording rotating field magnetostrictometer [Hoekstra et al., 1977] that consists primarily of a strain gage bridge, rotating permanent magnets, and a lock-in amplifier. The permanent magnets rotate at a frequency of about $30 \mathrm{~Hz}$, and the magnetostriction is detected at twice this frequency. Magnetostriction is recorded continuously as the sample is slowly heated and cooled in an inert atmosphere.

\section{THEORY}

In cubic crystals, the linear magnetostriction at saturation in an arbitrary direction is adequately described by a two constant expression [e.g. Cullity, 1972] given by 


$$
\begin{aligned}
& \lambda=\frac{3}{2} \lambda_{100}\left(\alpha_{1}^{2} \beta_{1}^{2}+\alpha_{2}^{2} \beta_{2}^{2}+\alpha_{3}^{2} \beta_{3}^{2}-\frac{1}{3}\right) \\
& +3 \lambda_{111}\left(\alpha_{1} \alpha_{2} \beta_{1} \beta_{2}+\alpha_{2} \alpha_{3} \beta_{2} \beta_{3}+\alpha_{1} \alpha_{3} \beta_{1} \beta_{3}\right)
\end{aligned}
$$

where $\lambda$ is the strain measured in the direction defined by direction cosines $\beta_{i}$ and $\alpha_{i}$ are the direction cosines of the magnetization. Both $\beta_{i}$ and $\alpha_{i}(i=1,2,3)$ are measured relative to the principal cubic axes. The magnetostriction constants along the $<100\rangle$ and $<111>$ crystal directions are $\lambda_{100}$ and $\lambda_{111}$, respectively. Equation (1) was derived under the assumption that the magnetostriction in a particular direction is the strain undergone by the crystal between an ideally demagnetized initial state with a random distribution of domain to magnetic saturation [e.g., Cullity, 1972]

The magnetostriction constants in equation (1) can be determined experimentally from measurements in a $\{110\}$ plane. Within this plane, let $\theta$ be the angle between $<001>$ and the direction of magnetization and $\varphi$ the angle between $<001>$ and $\lambda$. Equation (1) becomes

$$
\begin{aligned}
\lambda(\theta)=\frac{3}{4} & {\left[\lambda_{100} \cos ^{2} \varphi-\frac{1}{2}\left(\lambda_{111}+\lambda_{100}\right) \sin ^{2} \varphi\right] \cos 2 \theta } \\
& +\frac{1}{4} \lambda_{111} \sin 2 \varphi \sin 2 \theta+\text { const }
\end{aligned}
$$

The constant term in equation (2) is independent of direction and is related to the state of strain in the demagnetized state (unimportant for our analysis). We assume the magnetic field is large enough to align the magnetization along it. In our experiments, the field is rotated at a constant frequency $\omega$ in the plane of a disk shaped sample. The shape anisotropy of the disk aids the field in keeping the sample saturated. Equation (2) can be simplified for strains measured along arbitrarily chosen crystallographic directions in the $\{110\}$ plane. Thus for $\lambda$ parallel to [100], [111], or [110], respectively, and ignoring the constant term, we derive

$$
\begin{gathered}
\lambda(2 \omega t)=\frac{3}{4} \lambda_{100} \cos 2 \omega t \\
\lambda(2 \omega t)=\frac{3}{4} \lambda_{111} \cos 2 \omega t \\
\lambda(2 \omega t)=\frac{3}{8}\left(\lambda_{111}+\lambda_{100}\right) \cos 2 \omega t
\end{gathered}
$$

For polycrystalline samples with no preferred grain orientations, the linear strain is $\lambda(\theta)=3 / 2 \lambda_{2}\left(\cos ^{2} \theta-1 / 3\right)$, where $\lambda_{2}$ is the polycrystalline magnetostriction constant. This can be rewritten in terms of $2 \omega t$, so that

$$
\lambda(2 \omega t)=\frac{3}{4} \lambda, \cos 2 \omega t
$$

The polycrystalline magnetostriction constant $\lambda_{1}$ can also be given in terms of a linear combination of $\lambda_{100}$ and $\lambda_{111}$ by averaging equation (1) over all $\theta$ and $\varphi$ [e.g., Cullity, 1972]. If stress is uniform throughout the aggregate, then $\lambda_{2}=2 / 5 \lambda_{100}+3 / 5 \lambda_{111}$. This expression can be used to correlate magnetostriction results between polycrystalline and single-crystal samples.

In the strain gage technique, strain differences are measured between two well defined states. However, equations (1) to (6) were derived under the assumption that the demagnetized state of strain is known. Because this initial state is dependent upon domain structure, it can never be known unambiguously. To measure magnetostriction constants without any uncertainty regarding the initial demagnetized state, strain measurements are made between magnetically saturated states of magnetization [e.g.,

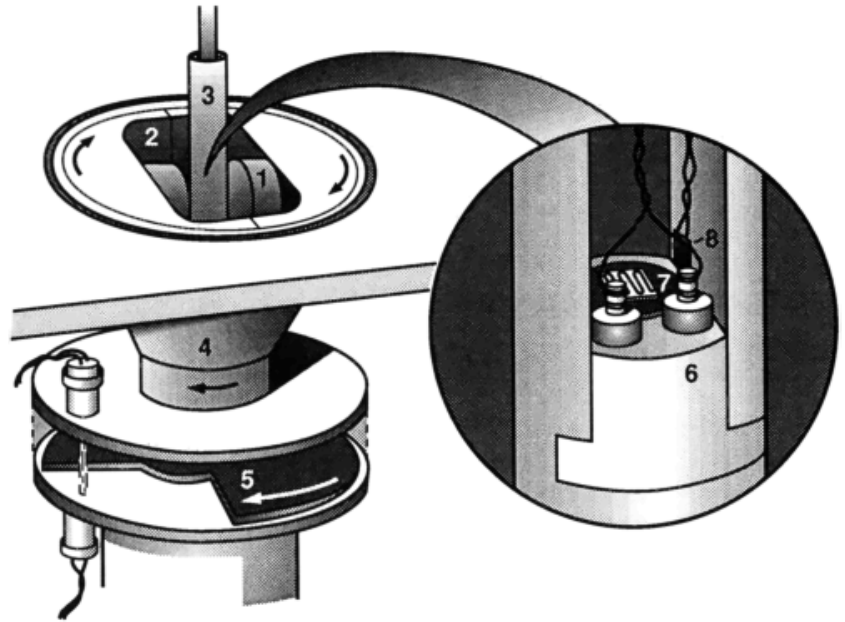

Fig. 1. Detail of the recording rotating field magnetostrictometer showing (1) $\mathrm{SmCO}_{3}$ permanent magnets, (2) rectangular steel frame, (3) furnace, (4) cone-shaped aluminum frame, (5) two hole light chopper, (6) brass sample holder, (7) sample with strain gage attached, and (8) platinum-resistance thermometer. Note in cut out view how the twisted wire pair is connected at the contact posts. The quartz dewar is not shown.

Cullity, 1972]. This is easily accomplished by measuring the strain when the magnetization is parallel $\left(\lambda_{1}\right)$ and perpendicular $\left(\lambda_{1}\right)$ to the strain gage. The saturation magnetostriction constant is then $2 / 3\left(\lambda,-\lambda_{\perp}\right)$, independent of initial domain configuration. In our experimental setup with a rotating field, the amplitude of the periodic signal is proportional to $\lambda_{r} \lambda_{1}$, which is also proportional to the second harmonic terms given by equations (4)-(6).

\section{EXPERIMENTAL PROCEDURES}

\section{Magnetostrictometer}

Magnetostriction is measured with a recording rotating field magnetostrictometer [Hoekstra et al., 1977]. The main parts of the magnetostrictometer are shown in Figure 1. The magnet assembly consists of a rectangular steel frame situated inside a cone-shaped aluminum frame. Two $\mathrm{SmCO}_{5}$ permanent magnets (diameter 2.5 cm and thickness $1 \mathrm{~cm}$ ) form the pole pieces with a $3-\mathrm{cm}$ gap giving a field at the center of approximately $170 \mathrm{mT}$. The magnets are mounted on the axis of a constant-speed dc motor that can rotate the magnets at constant frequency up to $30 \mathrm{~Hz}$. A two-hole light chopper is mounted directly below the magnet. The sample probe consists of a long aluminum tube, brass sample holder, resistance furnace, and a platinum resistance thermometer placed near the sample. The sample probe with sample attached is placed inside a quartz dewar and the whole assembly is situated between the pole pieces of the magnet. The sample is slowly heated and cooled $\left(10^{\circ} \mathrm{C} / \mathrm{min}\right)$ in $\mathrm{N}_{2}$ gas to reduce the effects of oxidation.

A metal strain gage is used to measure magnetostriction. The change in gage resistance $\Delta R_{g}$ is related to the change in length of the sample along the gage axis by the equation

$$
\frac{\Delta R_{g}}{R_{g}}=g \frac{\Delta l}{l}
$$

where $g$ is the gage factor and $R_{g}$ is the gage resistance. Depending on the particular gage, the temperature dependence of both $g$ 


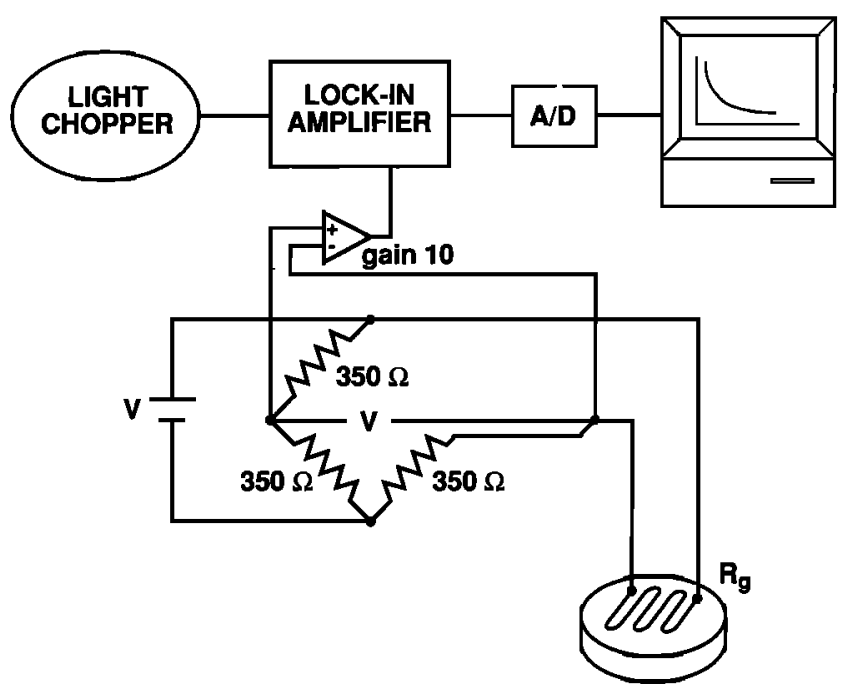

Fig. 2. Schematic circuit diagram of the measurement bridge. The quarter bridge consists of three $350 \mathrm{ohm}$ resistors and the strain gage $\left(R_{2}\right)$. The bridge excitation voltage is $V(=4.98 \mathrm{~V})$ and the output is $\Delta V$.

and $\boldsymbol{R}_{g}$ can be significant and must be taken into account. The strain gage forms one arm of a Wheatstone bridge circuit using a quarter-bridge configuration (see Figure 2). All three bridge resistors $\left(R_{1}=R_{2}=R_{3}=R_{b}\right)$ are hermetically sealed to reduce thermal drift, resulting in thermal coefficients of $1 \mathrm{ppm} /{ }^{\circ} \mathrm{C}$.

A reference signal with frequency $2 \omega$ is provided by a two-hole light chopper. A change in the position of the magnets with respect to the gage axis produces a change in resistance $\Delta R_{g}$, which in turn produces an unbalanced voltage $\Delta V$ across the bridge (Figure 2). The bridge output is periodic due to the rotating field and a lock-in amplifier measures the ac voltage at frequency $2 \omega$ [Hoekstra et al., 1977]. The dc output of the lock-in amplifier is a rms full-wave rectified signal that is proportional to the difference between the gage resistances with the field parallel and perpendicular to the gage axis. The advantage of using a lock-in amplifier is that it can select out any one frequency and measure it independently of other frequencies. The outputs from the lock-in amplifier and the platinum resistance thermometer are digitized and then processed by computer.

The sensitivity of the lock-in amplifier is $5 \mathrm{nV} / \sqrt{\mathrm{Hz}}$, which corresponds to maximum sensitivity of $2 \times 10^{-8}$ for $\lambda$ for a rotation frequency of $23 \mathrm{~Hz}$. The sensitivity of the system was determined to be approximately $200 \mathrm{nV}$ by measuring an $\mathrm{Al}_{2} \mathrm{O}_{3}$ sample. This voltage is equivalent to a measurement sensitivity of $\lambda=1 \times 10^{-7}$. Measurement uncertainty was estimated to be about $\pm 3 \%$.

In order to check that the $170 \mathrm{mT}$ field produced by the permanent magnets was sufficient to saturate the samples, the angular dependence of $\lambda(\theta)$ at room-temperature was determined by rotating the permanent magnets manually and recording the strain at every $10^{\circ}$ with a microvoltmeter instead of the lock-in amplifier. If a sample is not magnetically saturated, the rotational curve will contain odd harmonic terms. An extreme example of undersaturation may even produce a quasi-triangular waveform [e.g., Klerk et al., 1997]. Values of $\lambda$ were calculated from measurements made $90^{\circ}$ apart and using the equation $\lambda=2 / 3\left(\lambda_{r}-\lambda_{1}\right)$.

\section{Strain Gages}

Two types of metal foil strain gages were used depending on the maximum temperature of measurement: (1) Micromeasurement
(MM) type WK-06-031CF-350 (Ni-Cr alloy, $g=2.01 \pm 1.0 \%$ and $R_{G}=350.0 \pm 1.4$ ohms, room temperature values specified by manufacturer) for temperatures below $400^{\circ} \mathrm{C}$ and (2) Hi-Tech (HT) type HFP-12-125-SPW (Pt-W alloy, $g=3.62 \pm 2.0 \%$ and $R_{G}=$ $120 \pm 2.0 \mathrm{ohms}$, room temperature values specified by manufacturer) for temperatures above $400^{\circ} \mathrm{C}$. The active area of the gages was selected to be less than the area of the sample.

The HT gages were used for the pure magnetite samples whereas the MM gages were used for the titanomagnetite samples. Gages were bonded to flat surfaces with ceramic cement and cured at $200^{\circ} \mathrm{C}$ for 2 hours in a vacuum furnace. The temperature dependence of $g(T)$ was supplied by the manufacturers. The MM gage resistance was temperature independent but the HT gage resistance was not. The HT gage had to be further annealed at the maximum test temperature $\left(600^{\circ} \mathrm{C}\right)$ for $10 \mathrm{~min}$ according to the manufacturer's specifications. The annealing was necessary to compensate accurately for $R_{g}(T)$ and to cure the bonding cement for maximum stability. The annealing was carried out with the gage bonded to the sample in a flow of $\mathrm{N}_{2}$ to reduce the effects of oxidation.

\section{Sources of Noise}

Besides magnetostrictive strains, thermal stresses from heating and cooling, ambient temperature drift causing a change in resistance of the bridge resistors, magnetoresistance of the gage, induced electromagnetic noise, and poor electrical contact at high temperature can all produce spurious signals. By rotating the field and not the sample, any strains produced by thermal expansion of the sample and gage are not periodic in $2 \omega$ and therefore do not contribute to $\Delta R_{g}$. Also by rotating the field at $23 \mathrm{~Hz}$, a single measurement of $\Delta R_{g}$ can be made in less than $100 \mathrm{~ms}$. This reduces any thermal drift in the bridge resistors caused by ambient temperature fluctuations during a single measurement. Apparent strain produced by magnetoresistance above room temperature is negligible for MM gages [Hoekstra et al., 1977]. The HG gage is nonmagnetic and its magnetoresistance is assumed to be unimportant above room temperature.

For measurements below $400^{\circ} \mathrm{C}$, the most serious source of noise is from voltage induced by the rotating field in loops formed by the gage and lead wires. This noise can be reduced by carefully twisting the lead wires as close as possible to the contact posts, as shown in Figure 1 [Hoekstra et al., 1977].

For high-temperature measurements on magnetite, it was not possible to form twisted pairs of wire as close to the contact posts as was possible for measurements below $400^{\circ} \mathrm{C}$. Therefore, noise from induced emf is roughly 2 to 3 times higher for measurements on magnetite. However, the major experimental problem with the magnetite measurements is the difficulty of attaching and then maintaining electrical contact of the strain gage leads to the contact posts when temperatures exceed about $400^{\circ} \mathrm{C}$. The strain gages are small, extremely delicate and, easily damaged and a reliable and easy method of electrical connection has yet to be found. What this means is that at a certain temperature, usually between $350^{\circ}$ and $550^{\circ} \mathrm{C}$, contact is broken or short circuited and the signal goes to zero. Reconnection is possible once the sample is cooled to room temperature, but each time this oocurs there is the potential of damaging the strain gage. Despite these problems, we were able to obtain some results up to $580^{\circ} \mathrm{C}$ for polycrystalline magnetite. However, these results are of much poorer quality than those obtained for the titanomagnetite samples. 
Samples

Magnetostriction was measured on synthetic polycrystalline samples and natural and synthetic single crystals. Polycrystalline samples of $\mathrm{Fe}_{3} \mathrm{O}_{4}$ (TMO), $\mathrm{Fe}_{2.6} \mathrm{Ti}_{0.4} \mathrm{O}_{4}$ (TM40), and $\mathrm{Fe}_{2.4} \mathrm{Ti}_{0.6} \mathrm{O}_{4}$ (TM60) were synthesized from mixtures of analytical grade $\mathrm{Fe}_{2} \mathrm{O}_{3}$ and $\mathrm{TiO}_{2}$. The starting material was pressed into pellets' and then suspended in a vertical tube furnace at $1300^{\circ} \mathrm{C}$ for 48 hours in a mixture of $\mathrm{CO}-\mathrm{CO}_{2}$ to control oxygen fugacity. Values of $\mathrm{fO}_{2}$ (in atm) were $10^{-6.0}$ (TM0), $10^{8.0}$ (TM40), and 10 $0^{-9.0}$ (TM60) and could be controlled to within $0.1 \mathrm{log}$ units of $\mathrm{fO}_{2}$. After sintering, the pellet was dropped directly into liquid nitrogen and quenched rapidly. A polycrystalline sample of $\mathrm{Fe}_{22} \mathrm{Al}_{0.1} \mathrm{Mg}_{0.1} \mathrm{Ti}_{0.6} \mathrm{O}_{4}$ (AMTM60) was also measured [Moskowitz et al., 1988]. The results of optical, $X$ ray powder diffraction, thermomagnetic, and electron microprobe analyses indicated that all samples were single phase within the accuracies of the analytical methods. Magnetic moment was measured with a vibrating sample magnetometer (VSM) with a maximum field of $1.5 \mathrm{~T}$. Characteristic properties are given in Table 1 . Samples were cut into disks approximately $10 \mathrm{~mm}$ in diameter and $1-2 \mathrm{~mm}$ thick for the magnetostriction measurements. The absolute magnitude of $\lambda_{3}$ for sintered polycrystalline samples may be lower than for single crystals due to porosity, but the relative compositional and thermal dependence of $\lambda_{\mathrm{r}}$ should be unaffected for a series of samples produced under identical conditions.

Magnetostriction of two single crystals of pure magnetite was also measured. One was a natural single crystal from Magnet Cove, Arkansas, and the other was a synthetic crystal supplied by M. Young of the Lawrence Livermore National Laboratory. The synthetic crystal was grown in the $<110\rangle$ direction by the floatingzone technique in an arc image furnace. The natural and synthetic crystals were cut into $\{110\}$ disks approximately $10 \mathrm{~mm}$ in diameter and 1-2 mm thick. Strain gages were cemented parallel to either the $\langle 100\rangle$ or $\langle 111\rangle$ direction on individual samples.

Unfortunately, none of the high temperature measurements for the single crystal samples were satisfactory. The main reason for the lack of success was the electrical connection problem referred to earlier. After many failed attempts it was decided to conserve the remaining single crystals until a reliable connection technique is developed.

\section{RESULTS}

\section{Room Temperature Measurements}

Room temperature values of $\lambda_{100}$ and $\lambda_{111}$ for single-crystal samples and $\lambda_{\text {}}$ for polycrystalline samples are given in Table 2 . The single-crystal results of magnetite are in excellent agreement
TABLE 2. Room Temperature $\left(23^{\circ} \mathrm{C}\right)$ Values of iMagnetostriction Constants

\begin{tabular}{lll}
\hline Composition & \multicolumn{1}{c}{$\begin{array}{c}\text { Polycrystal, } \\
\lambda_{\mathrm{r}} \times 10^{-6}\end{array}$} & $\begin{array}{c}\text { Single Crystal, } \\
\lambda \times 10^{-6}\end{array}$ \\
\hline TM40 & $122.7(10)$ & $\ldots$ \\
TM60 & $1113(8)$ & $\ldots$ \\
AMTM60 & $47.2(4)$ & $\ldots$ \\
TM0A & $39.4(6)$ & $\ldots$ \\
TM0B & 47.1 & $\ldots$ \\
TM0[111] & $\ldots$ & 78.2 \\
TM0[100] & $\ldots$ & $-21.8(12)$ \\
\hline
\end{tabular}

TMOA and TMOB refer to the same sample but are treated separately for magnetostriction results (see text for details). TMO[111] refers to the natural single crystal from Magnet Cove, Arkanas, measured in the [111] direction. TMO[100] refers to the synthetic crystal measured in the [100] direction. Numbers in parentheses refer to standard deviation in least significant units.

with those of previous investigations. Room temperature values of $\lambda_{\text {f }}$ for the polycrystalline samples of TM0, TM40, and TM60 also agree with previously published values calculated from single crysta! results [Syono, 1965; Klerk et al., 1977]. The close agreement between polycrystal and single-crystal data verifies that $\lambda_{\text {, }}$ was measured accurately for sintered polycrystalline samples with the magnetostrictometer. In addition, the good agreement between our results and those previously published confirms that the magnetic field produced by the permanent magnets was sufficient to saturate the samples in the plane of the disk. This was further confirmed by two additional experiments. First, the rotational curve of $\lambda(\theta)$ for each sample at room temperature was measured and all were found to contain only $2 \theta$ sinusoidal components within experimental error. An example is shown in Figure 3. Second, $M-H$ curves were measured in fields up to $1500 \mathrm{mT}$. The magnetic moment of each sample at $170 \mathrm{mT}$ (the field within the gap of the permanent magnets) was within $95 \%$ of the moment at $1500 \mathrm{mT}$.

\section{High-Temperature Measurements: Polynomial Fits}

Titanomagnetites. The variation of magnetostriction with temperature, measured at $1^{\circ} \mathrm{C}$ intervals between room temperature and the Curie temperature, was measured three times for each titanomagnetite sample. The results are shown in figures 4 to 6 . For all runs the results were found to be reversible with heating and cooling and reproducible between different runs. Average values of $\lambda_{3}(T)$ were calculated by first averaging the two magnetostriction values at each temperature along the heating and

TABLE 1. Characteristic Properties of Synthetic Polycrystalline Titanomagnetites

\begin{tabular}{|c|c|c|c|c|}
\hline Composition & $T_{c},{ }^{\circ} \mathrm{C}$ & $\begin{array}{c}M_{s}\left(23^{\circ} \mathrm{C}\right) \\
\mathrm{Am}^{2} \mathrm{~kg}^{-1}\end{array}$ & $\begin{array}{c}\text { Cell Parameter, } \\
\text { nm }\end{array}$ & $\begin{array}{c}\text { Mole Percent } \\
\mathrm{Fe}_{2} \mathrm{TiO}_{4} \\
\end{array}$ \\
\hline TMO & 573 & 91.7 & $0.8395(2)$ & 0.00 \\
\hline TM40 & 304 & 48.2 & $0.8449(1)$ & $39.68(69)$ \\
\hline TM60 & 142 & 27.4 & $0.8479(2)$ & $59.69(61)$ \\
\hline AMTM60 & 73 & 17.2 & $0.8469(2)$ & $60.59(87)$ \\
\hline
\end{tabular}

Curie temperatures determined by the method of thermodynamic coefficients, $M_{\text {, determined using equation }}$ (A3) in the appendix, cell parameter determined by a least squares refinement program, and mole percent $\mathrm{Fe}_{2} \mathrm{TiO}_{4}$ determined by electron microprobe analysis for 50 individual spots based on $\mathrm{R}_{3} \mathrm{O}_{4}$ stoichiometry. Numbers in parentheses refer to standard deviation in least significant units. 


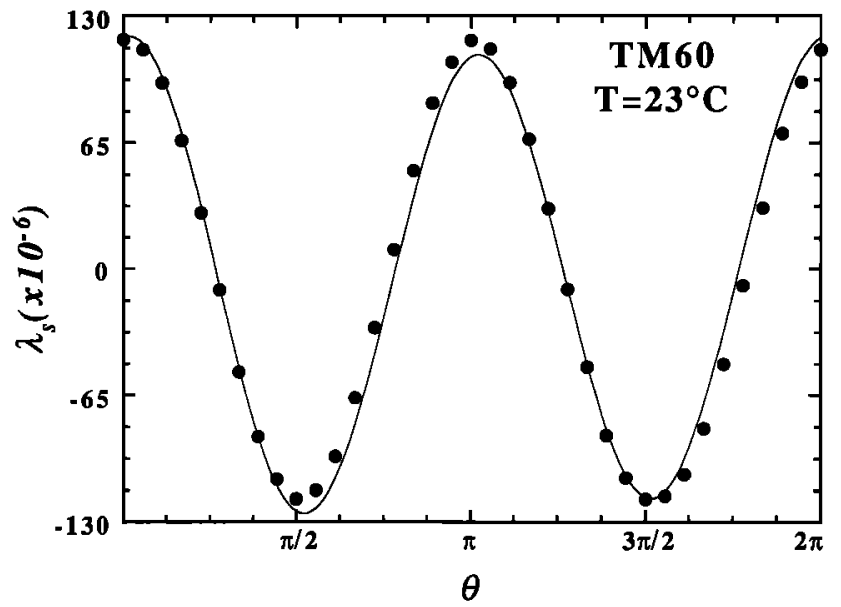

Fig. 3. The angular dependence of $\lambda(\theta)$ for TM60 at room temperature. This curve was determined by rotating the permanent magnets manually and recording the strain at $10^{\circ}$ intervals with a microvoltmeter instead of the lock-in amplifier.

cooling curve. This was done for each of the three separate runs and then all three runs were averaged to calculate the final average curve. The average $\lambda_{3}(T)$ data for each sample was fit by a fifth-order polynomial by the method of least squares. A fifthorder curve was used not because it was statistically justified but because it can be used to reproduce the experimental results reasonably well. The curve fits are plotted for each sample in Figures 4 to 6 . The six polynomial coefficients are given in the Table 3.

Magnetite. As discussed previously, due to experimental difficulty the magnetite data are of much poorer quality than the titanomagnetite results. This is evident in Figure 7, which shows the results of our best runs. The data are from the same polycrystalline sample measured several times in sequence from run 1 to 12. The lower series of curves (denoted as TMOA) are for runs $1,2,3,6$, and 8 and show only the heating portions of the curves. The maximum temperature of measurement before the signal was lost was $480^{\circ} \mathrm{C}$ for run 3 . Room temperature values of

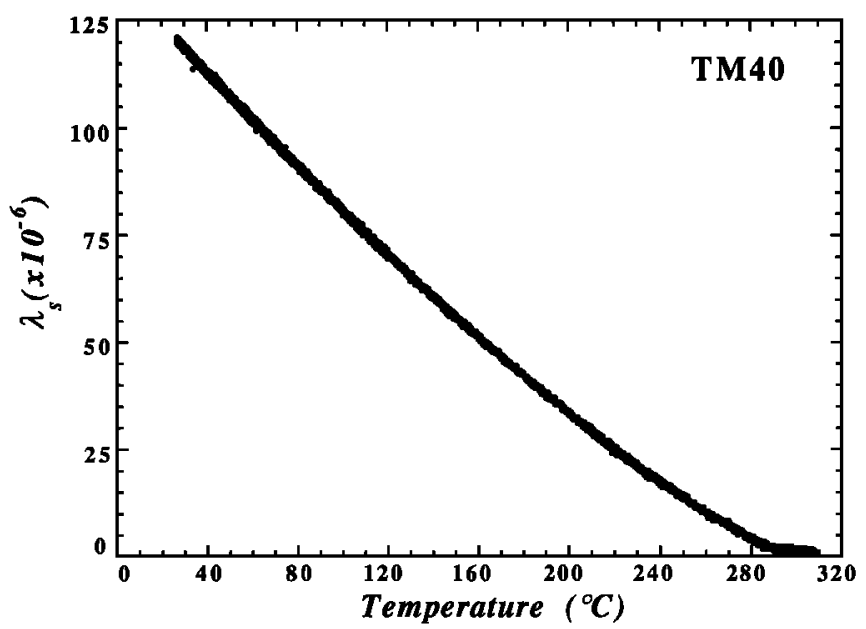

Fig. 4. Magnetostriction as a function of temperature for TM40. The results include the heating and cooling curves of three separate runs. The curve describing the best fit fifth-order polynomial is hidden by the densely spaced data.

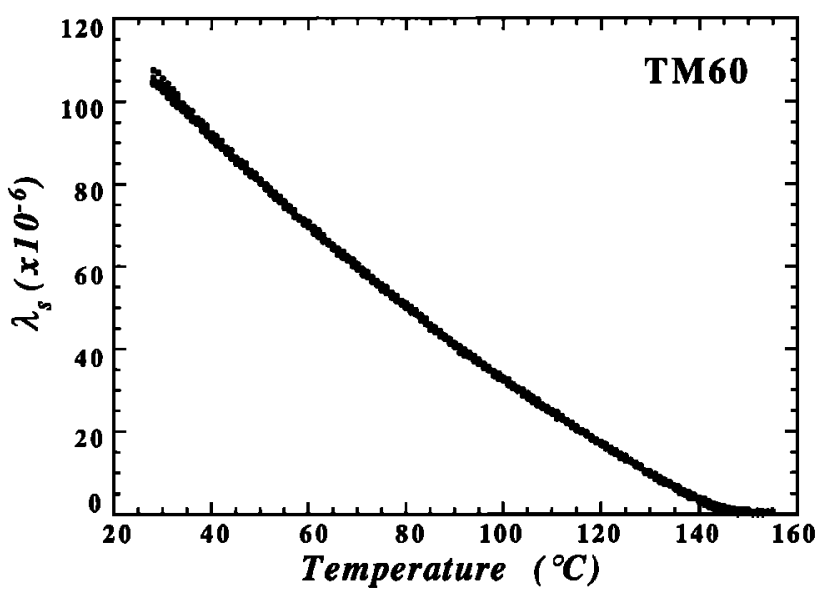

Fig. 5. Magnetostriction as a function of temperature for TM60. The results include the heating and cooling curves of three separate runs. The curve describing the best fit fifth-order polynomial is hidden by the densely spaced data.

$\lambda_{3}$ for the five runs were approximately $40 \times 10^{-6}$, in good agreement with literature values. However, after run 8 , the observed values of $\lambda_{2}$ unexpectedly and irreversibly increased by approximately $15 \%$ at room temperature. The reason for this is not known but could be related to the aging of the gage, the ceramic bond, or both, with repeated thermal cycling. Any change in the resistance of the gage lead wires due to degradation or kinks may also produce an apparent increase in magnetostriction. Nevertheless, two later runs, 11 and 12 (denoted as TMOB), yielded roughly reversible and reproducible data up to $540^{\circ} \mathrm{C}$. Above $540^{\circ} \mathrm{C}$, only the heating portion of run 11 provided results to $580^{\circ} \mathrm{C}$.

Average values of $\lambda_{3}(T)$ for TMOA and TMOB runs were calculated by averaging the values at each temperature along the heating and, if available, the cooling curves. This was done for each of the separate runs, and then all runs within each series were averaged to form the final average curves. The average $\lambda_{s}(T)$ data fCr 'ГMOA and TMOB runs were fit by a fifth-order polynomial by the method of least squares. The results are shown in Figure 8 and the polynomial coefficients are given in Table 3. Aside from the offset at room temperature, the thermal dependence of $\lambda_{\text {, }}$

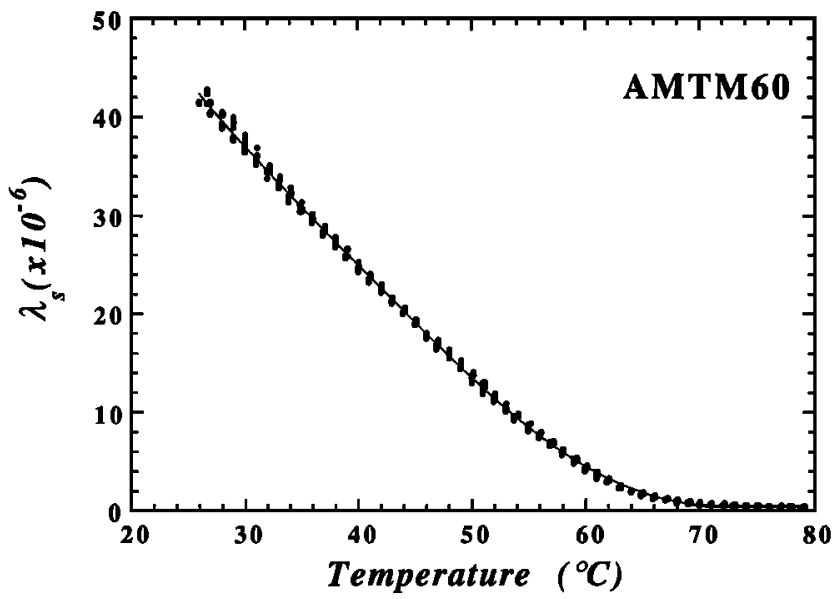

Fig. 6. Magnetostriction as a function of temperature for AMTMt0. The results include the heating and cooling curves of three separate runs. The solid line is the best fit fifth-order polynomial. 


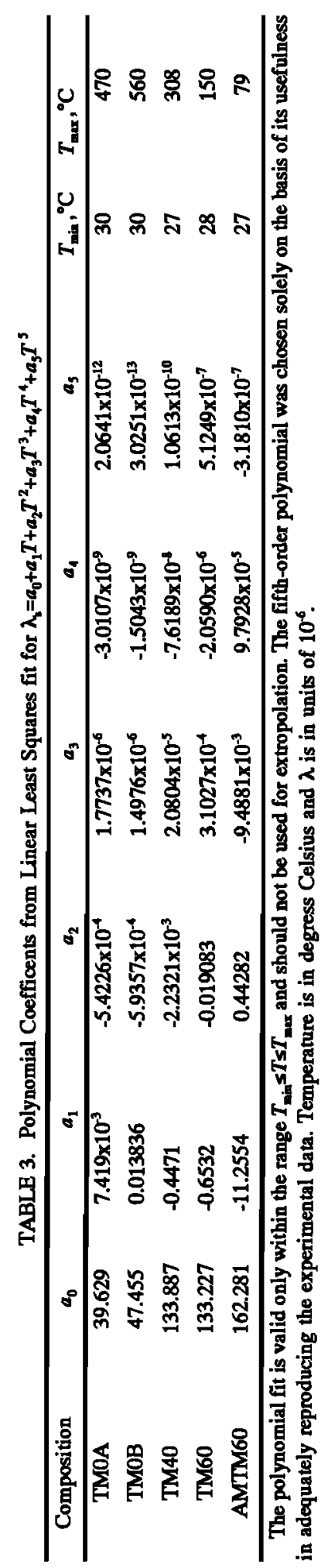

is similar for both TMOA and TMOB. However, the quality of the data is less than satisfactory and the results must be considered only as preliminary.

\section{High-Temperature Measurements: Power Law Fits}

Besides polynomial fits, it is common to fit the experimental thermal dependence of magnetic constants such as magnetostriction, magnetocrystalline, and magnetic exchange constants, to simple power law equations in terms of either the spontaneous or saturation magnetization, $M_{s}(T)$, or reduced temperature, $T / T_{c}$. There are two main reasons for fitting the experimental data to power law relations. The first and more fundamental reason is to determine the critical exponents in order to test various theoretical predictions about the physical origins of magnetostriction. The second is to determine empirical fits over a wide temperature range $\left(23^{\circ} \mathrm{C}\right.$ to $\left.T_{c}\right)$ in order to provide simple relations for modeling the thermal dependence of magnetic domains, coercivity, and remanence. For either case, the theoretical justification for a power law approximation is that as the temperature approaches the Curie temperature from below, magnetization and other magnetic parameters can be approximated as a power series expansion in $1-T / T_{c}$ near $T_{c}$. In the temperature range studied $\left(23^{\circ} \mathrm{C}\right.$ to $\left.T_{c}-10^{\circ}\right)$, the experimental data for magnetite and titanomagnetite can be fit to simple power law relationships, even though the temperature range extends significantly below the Curie temperatures. For pure magnetite, the temperature range over which a simple power law is valid $(T / T>0.35)$ is consistent with high temperature measurements of magnetocrystalline anisotropy and exchange [Fletcher and O'Reilly, 1974; Collins and Saunderson, 1970].

Although, in principle, fitting the experimental data to a simple power law should be an easy application of regression analysis, in practice, it proved otherwise. Besides random experimental errors, the following three factors, each subjective in nature, influenced the value of the exponent determined by the regression analysis: (1) the method used to calculate $M_{s}(T)$ from high field measurements of magnetization; (2) the choice of Curie temperature; and (3) the temperature interval used in the curve fitting. To reduce the subjective component, it was necessary to use a more complicated curve fitting procedure adapted from a method developed by Coe at al. [1978] and is discussed in the appendix. Also in the

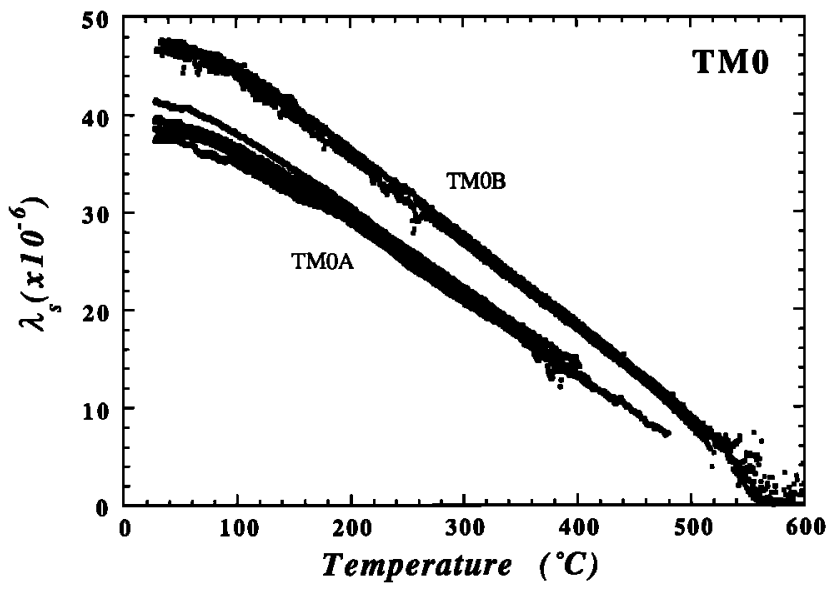

Fig. 7. Magnetostriction as a function of temperature for TMO. The results include the heating and cooling curves of several separate runs. Curves deisuied by TMOA. icclude runs $1,2,3,6$, and 8 , whereas curves decoted by TMOB include runs 11 and 12. Note that TMOA and TMOB refer to the same polycrystalline sample of magnetite but are treated separately due to the irreversible increase of $\lambda_{\boldsymbol{r}}$ after run 8 . 
appendix, I describe three methods for calculating $M_{s}(T)$ from high-field measurements and two methods for calculating $T_{\mathrm{c}}$. Most of the variability in relating magnetostriction to magnetization comes from different ways of calculating $M_{s}(T)$. The method used to calculate $M_{s}(T)$ is important because the results of the regression analysis are sensitive to the variation of $M_{s}(T)$ near $T_{c}$, where the magnetization changes the most rapidly. In the following analysis, $M_{s}(T)$ was calculated by the method of thermodynamic coefficients (see the appendix). Results obtained from all the different methods are summarized in the appendix.

The exponents in the power law equations were determined by two slightly different methods. First, and most straightforward, is a simple power law fit of $\lambda_{2}$ and $M_{2}$, namely,

$$
\lambda_{s}(T)=a_{1} M_{s}(T)^{n_{1}}
$$

where $a_{1}$ and $n_{1}$ are constants determined by the fit. This equation assumes that each $\lambda_{2} M_{1}$ pair corresponds to the same absolute temperature, or equivalently to the same value of $T / T_{c}$. In other words, there is no systematic difference between the absolute temperature measured by the magnetostrictometer and the VSM. It also assumes that $M_{s}(T)$ was accurately determined from the high-field measurements. The exponents determined in this fashion, using the best fit criteria of Coe et al. [1978], are given in the last column of Table 4.

The second method, described in the appendix, produces more consistent results between samples. First, $\lambda_{s}(T)$ and $M_{s}(T)$ are fit to equations of the form

$$
\begin{aligned}
& \lambda_{s}(T)=a_{2}\left[1-\frac{T}{T_{c}}\right]^{n_{2}} \\
& M_{s}(T)=a_{3}\left[1-\frac{T}{T_{c}}\right]^{n_{s}}
\end{aligned}
$$

where $a_{2}, a_{3}, n_{2}$, and $n_{3}$ are constants determined by the regression analysis, $T_{c}$ is the Curie temperature, and $T$ is absolute temperature. Since a simple linear least squares fit to equations (9) and (10) is dependent on a priori knowledge of $T_{c}$ a nonlinear least squares procedure that included $T_{c}$ as an adjustable parameter was used instead (see the appendix). This method, along with other considerations discussed in the appendix, provided a consistent estimate of the Curie temperature and exponents from both the $\lambda_{s}(T)$ and $M_{s}(T)$ data sets. Once $n_{2}$ and $n_{3}$ are estimated, the relationship between $\lambda_{s}(T)$ and $M_{s}(T)$ can be determined from

$$
\lambda_{s}(T) \propto M_{s}(T)^{\frac{n_{2}}{n_{3}}}
$$

By using equation (11), the assumption is made that the indepen-

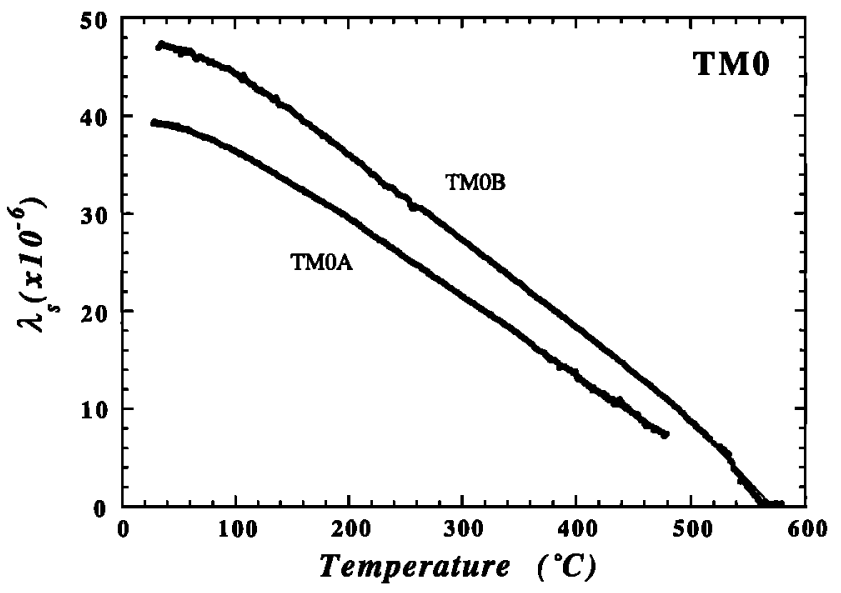

Fig. 8. Average curves of magnetostriction as a function of temperature for TMOA and TMOB based on the data in Figure 7. Due to the dense spacing of data, the best fit fifth-order polynomials is barely visible.

dent variable is the reduced temperature $T / T_{\mathcal{O}}$ even though the absolute value of $T_{c}$ inferred from the $\lambda_{c}$ and $M_{k}$ data may be different. Calculating exponents in this way avoids the problem of a systematic shift in temperature measured between the magnetostrictometer and the VSM. A small constant shift in temperature does not affect the exponents obtained from equations (9) and (10). The exponents determined from this second method of analysis are given in the columns 2-4 of Table 4 . The magnetostriction and magnetization data and the power law fits are shown in Figures 9 to 12 .

Figure 13 shows a bilogarithmic plot of $\lambda_{s}(T)$ against $1-T / T_{c}$ for the three titanomagnetite compositions and for magnetite. The solid lines are the best fitting curves calculated from equation (9). Over the entire temperature range from $25^{\circ} \mathrm{C}$ to approximately $T_{c}$ $10^{\circ} \mathrm{C}$, the thermal dependence of magnetostriction varies approximately as $\left(1-T / T_{c}\right)^{1.3}$ for titanium-rich titanomagnetite. In contrast, the results for magnetite suggest an exponent slightly less than 1.0 , with $\lambda_{\text {}}$ varying approximately as $\left(1-T / T_{c}\right)^{0.9}$. The tailing off in the experimental data near $T_{c}$ most likely reflects the noise threshold.

The absolute value of $\lambda_{\text {, for }}$ any given value of reduced temperature increases systematically with titanium content. This trend is consistent with the concomitant increase in the concentration of $\mathrm{Fe}^{2+}$ with titanium substitution. Octahedral $\mathrm{Fe}^{2+}$ has large spin orbit coupling and its increasing concentration in Ti-rich titano. magnetites is usually associated with increases in both magnetostriction and magnetocrystalline anisotropy [e.g., O'Reilly, 1984]. However, any formal analysis of the absolute values of magneto-

TABLE 4. Power Law Exponents for the Thermal Dependence of Magnetostriction and Magnetization.

\begin{tabular}{ccccc}
\hline Composition & $\begin{array}{c}\lambda \propto\left(T_{c}-T\right)^{\mathrm{n}} \\
n_{2}\end{array}$ & $\begin{array}{c}M_{s} \propto\left(T_{c}-T\right)^{n 3} \\
n_{3}\end{array}$ & $\begin{array}{c}\lambda \propto M_{s}{ }^{n 3 / n 2} \\
n_{3} / n_{2}\end{array}$ & $\begin{array}{c}\lambda \propto M_{s}{ }^{n 1} \\
n_{1}\end{array}$ \\
\hline TM0A & $0.90(1)$ & $0.38(1)$ & $2.37(6)$ & $2.66(1)$ \\
TM0B & $0.86(1)$ & $0.38(1)$ & $2.26(9)$ & $2.23(1)$ \\
TM40 & $1.29(1)$ & $0.37(1)$ & $3.49(10)$ & $3.78(2)$ \\
TM60 & $1.28(1)$ & $0.47(2)$ & $2.72(12)$ & $2.51(4)$ \\
AMTM60 & $1.29(2)$ & $0.46(6)$ & $2.80(34)$ & $3.86(7)$ \\
\hline
\end{tabular}

$M_{s}$ is determined by the method of thermodynamic coefficients using equation (A3) in the appendix. TMOA and TMOB refer to the same sample but are treated separately only for the magnetostriction results. Numbers in parentheses refer to standard deviation in least signific ant units. 


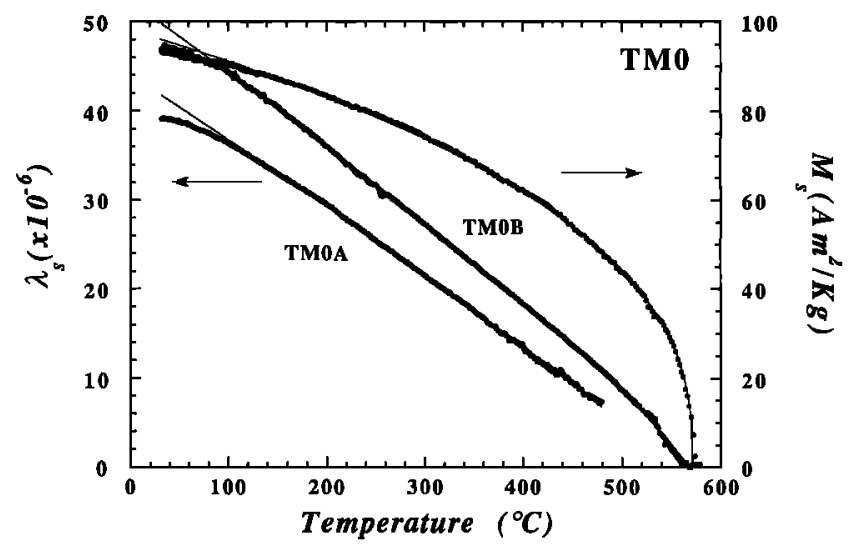

Fig. 9. Temperature dependence of magnetostriction and magnetization for TMO. Circles denote the experimental data and the solid lines denote the best fits to (10) and (11).

striction must be done with single-crystal data. Finally, the results show that $\lambda_{3}$ varies approximately as the third power of magnetization for Ti-rich titanomagnetites, in general agreement with the phenomenological single-ion theory of Callen and Callen [1963].

\section{Discussion}

The thermal dependence of magnetostriction for TM40, TM60, and AMTM60 was successfully measured between room temperature and the Curie temperature. In contrast, the magnetostriction results for magnetite were of a considerably poorer quality, particularly in the important temperature range above $500^{\circ} \mathrm{C}$, and must be viewed as preliminary at this stage. Although the accuracy of the magnetite results may be poor, they are consistent with the earlier single crystal measurements of Klapel and Shive [1974] that show an approximately linear decrease of $\lambda_{111}$ with temperature. Assuming that the new magnetostriction data for magnetite are reliable, the results show that with the addition of titanium, the thermal dependence of $\lambda_{s}(T)$ changes from roughly $\left(1-T / T_{c}\right)^{0.9}$ for TMO to $\left(1-T / T_{c}\right)^{1.3}$ for TM40 and TM60. Although the measurements done here cannot separate $\lambda_{111}$ and $\lambda_{100}$, the change in the thermal dependence of $\lambda_{3}$ with Ti substitution is consistent with the low-temperature (80-300 K) single-crystal measurements of

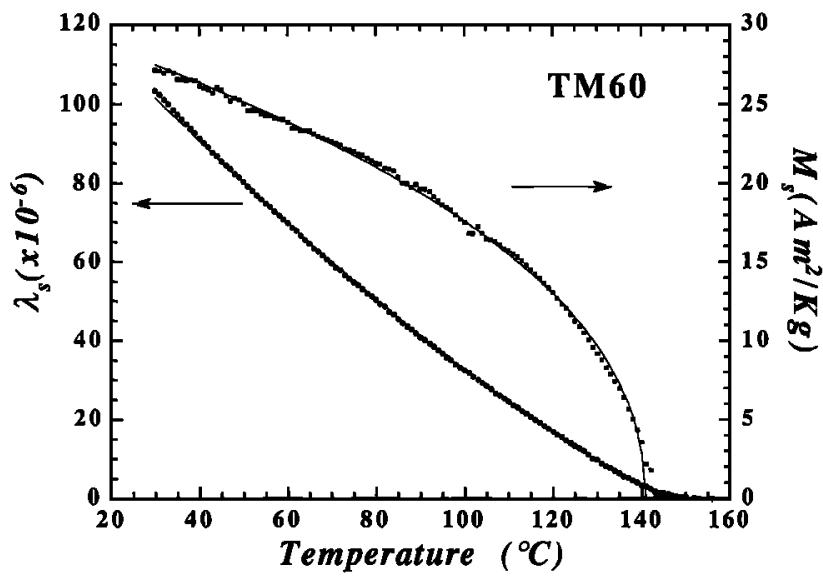

Fig. 11. Temperature dependence of magnetostriction and magnetization for TM60. Circles denote the experimental data and the solid lines denote the best fits to (10) and (11).

Syono [1965] and Klerk et al. [1977]. In both studies, Ti-rich compositions (TM40-TM100) show a much stronger temperature dependence for both constants, and most notably for $\lambda_{100}$, than for compositions near magnetite. It is reasonable to expect this behavior to extend to temperatures approaching the Curie temperature.

Although the separate behavior of $\lambda_{111}$ and $\lambda_{100}$ cannot be determined from the measurements of $\lambda_{s}(T)$, we can still make some general statements about $\lambda_{111}(T)$ and $\lambda_{100}(T)$. For example, the smooth decrease of $\lambda_{s}(T)$ with temperature and its positive only values for TM40, TM60, and AMTM60 (see Figures 4-6) suggest that $\lambda_{111}$ and $\lambda_{100}$ remain positive and that each constant monotonically decreases at roughly the same rate throughout the temperature range of measurement. A significantly different temperature dependence of either $\lambda_{111}(T)$ and $\lambda_{100}(T)$, or an increase in absolute value or even changes in sign of either constant for a certain temperature range, may produce "dips", "bumps", or compensation points $\left(\lambda_{2}=0\right)$ in the $\lambda_{s}(T)$ curve. The positive values of $\lambda_{1}$ for all temperatures for these compositions predict that the direction of tensile stress will be the easy axis of magnetization (i.e., $\left.\lambda_{s} \sigma<0\right)$.

The magnetite results also show only positive values and a smooth decrease in $\lambda_{s}(T)$ with temperature (see Figure 8). Klapel

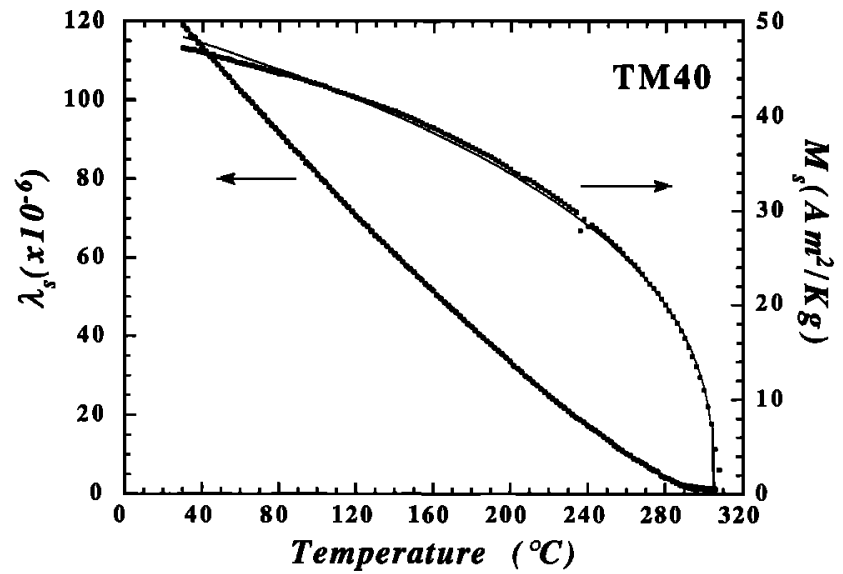

Fig. 10. Temperature dependence of magnetostriction and magnetization for TM40. Circles denote the experimental data and the solid-lines denote the best fits to (10) and (11).

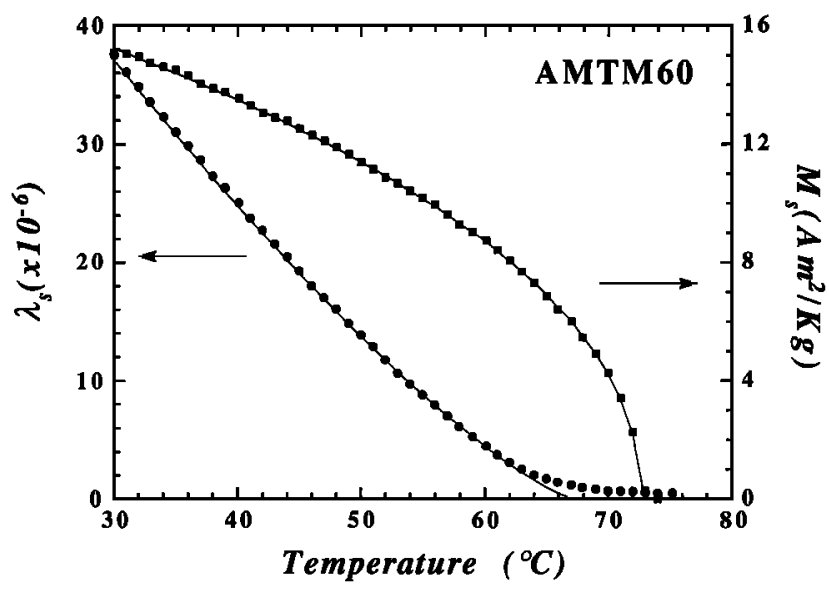

Fig. 12. Temperature dependence of magnetostriction and magnetization for AMTM60. Circles denote the experimental data and the solid lines denote the best fits to (10) and (11). 


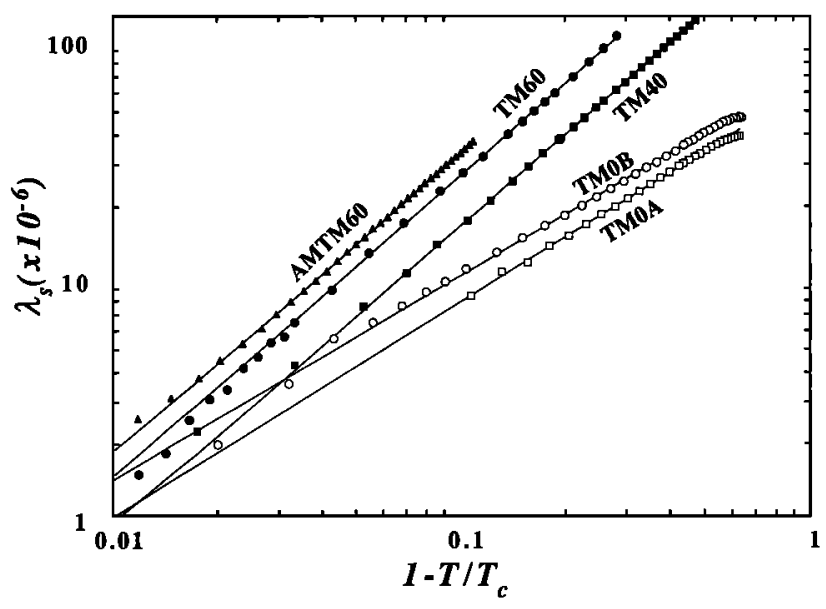

Fig. 13. Magnetostriction constant for TMOA, TMOB, TM40, TM60, and AMTM60 plotted against 1-T/T on a $\log -\log$ scale.

and Shive [1974] were skeptical about their $\lambda_{100}(T)$ data, which suggested an apparent increase in $\lambda_{100}$ between $100^{\circ}$ and $200^{\circ} \mathrm{C}$. An increase in $\lambda_{100}$ should produce a "dip" in the $\lambda_{s}(T)$ curve (i.e., $\left.\lambda_{100}<0\right)$. The new data suggest that $\lambda_{100}(T)$ does not increase initially with temperature but instead decreases monotonically with temperature. My results also suggest that $\lambda_{111}(>0)$ and $\lambda_{100}(<0)$ do not change sign over the temperature range up to $540^{\circ} \mathrm{C}$. The precision of the data above $540^{\circ} \mathrm{C}$ is inadequate to predict any sign changes in $\lambda_{111}$ and $\lambda_{100}$.

The magnetostriction results can be used to predict qualitatively the temperature dependence of the first magnetocrystalline anisotropy constant $K_{1}$ using a simple scaling law approximation. There are no experimental measurements of $K_{1}(T)$ above room temperature for any titanomagnetites except those close in composition to magnetite [Fletcher and O'Reilly, 1974]. Thus, for theoretical micromagnetic calculations for TM60, $K_{1}(T)$ is assumed to have the same dependence as magnetite, namely, $K_{1} \propto M_{2}^{8}$, [e.g., Moskowitz and Halgedahl, 1987; Halgedahl, 1987; Moon, 1990]. Alternatively, because of the common origin of magnetocrystalline anisotropy and magnetostriction, I have assumed that there exists a simple linear relationship between the power law exponents for $K_{1}(T)$ and $\lambda_{3}(T)$ throughout the titanomagnetite series. This is based on the single-crystal titanomagnetite data of Syono [1965] and Klerk et al. [1977] which suggests that within the temperature range studied (80-300 K), the temperature dependence of $K_{1}(T), \lambda_{111}(T)$, and $\lambda_{100}(T)$ are related in some fashion and correlated with titanium content. Although a reasonable approximation, a linear relationship between exponents has as yet no theoretical justification other than general considerations regarding scaling law relations among critical exponents near phase transitions [e.g., Stanley, 1971]. Despite this shortooming, one can use such a linear approximation along with the magnetostriction results for TM0 and TM60 (see Table 4) to predict that $K_{1}(T)$ for TM60 should decrease with temperature as $M_{s}(T)^{10}$. This temperature dependence is much steeper than that observed in magnetite. If true, magnetocrystalline contributions to remanence, coercivity, and the thermal dependence of domains in TM60 (TM40 and AMTM60 as well) should be even less important than previously believed.

Finally, we can compare the magnetostriction results for AMTM60 with the experimental data of Halgedahl [1991] for the thermal dependence of domain widths for the same composition and estimate the approximate thermal dependence of the exchange constant, $A(T)$, for AMTM60. The exchange constant is an import- ant parameter for micromagnetic domain calculations and one that has been experimentally determined only for magnetite.

Halgedahl [1991] measured the domain widths of maze like patterns in AMTM60. Making certain assumptions about the validity of the domain observations, assuming that the thermal dependence of $A(T)$ in AMTM60 is identical to that experimentally determined for magnetite $\left(A(T) \propto M_{s}(T)^{1.6-18}\right.$, see Moskowitz and Halgedahl [1987] and Heider and Williams [1988]), and using the Kittel-type model [see Halgedahl, 1987; Moskowitz and Halgedahl, 1987], Halgedahl [1991] estimated an upper limit of $\lambda_{s}(T) \propto M_{s}(T)^{26-3 . A}$. This thermal dependence is virtually identical to the one measured here for AMTM60 (see Table 4). We can turn this argument around and estimate directly the thermal dependence of the exchange constant now that we know the thermal dependence of magnetostriction. Our results for AMTM60 predict that $A(T) \propto M_{s}(T)^{1.6-22}$ and is indeed similar to magnetite as hypothesized. Furthermore, if the real thermal dependence of $A(T)$ is not too different from our predicted dependence, the magnetostriction data justify some of the major assumptions used by Halgedahl [1991] in her analysis of maze like domain pattems in AMTM60. Most notably, that (1) the maze like patterns represent "global energy minimum" (GEM) states during cooling; (2) any thermal changes in domain wall widths have negligible effects on domain widths in GEM states; and (3) the simple Kittel-like model works remarkably well for describing the thermal dependence of GEM states in domain patterns dominated by stress.

\section{CONCLUSIONS}

1. The thermal dependence of $\lambda_{s}(T)$ in the range $23^{\circ} \mathrm{C}$ to $T_{c}-$ $10^{\circ} \mathrm{C}$ varies as $\left(1-T / T_{c}\right)^{13}$ for Ti-rich titanomagnetites TM40, TM60, and AMTM60. For pure magnetite, the thermal dependence is almost linear with temperature, varying as $\left(1-T / T_{c}\right)^{0.86-0.90}$.

2. The polycrystalline magnetostriction constant is well approximated by a power law equation of the saturation magnetization, $\lambda_{s}(T) \propto M_{s}(T)^{\mathrm{n}}$, where $n=2.31$ (TM0), 3.47 (TM40), 2.75 (TM60), and 2.80 (AMTM60). The variation in the value of $n$ with composition is primarily a result of the variation in the temperature dependence of saturation magnetization with composition.

3. Due to experimental difficulty, the magnetostriction results for magnetite should be viewed with caution and considered only as preliminary at this point. Despite the problems, however, our measurements for magnetite are in good agreement with those obtained by Klapel and Shive [1974].

4. The magnetostriction results for AMTM60 are in excellent agreement with the theoretical prediction of Halgedahl [1991] based on the experimental observations of the thermal dependence of domain widths in AMTM60.

5. Also, using the observations of Halgedahl [1991] our results predict that the thermal dependence of the exchange constant in AMTM60 varies as $M_{s}(T)^{1,6-22}$.

6. Applying a scaling law relation between power law exponents, the magnetostriction results qualitatively predict that $K_{1}(T) \propto M_{\mathrm{s}}{ }^{10}$ in Ti-rich titanomagnetites. This predicted temperature dependence is greater than that observed for magnetite.

\section{APPENDIX}

\section{Temperature Dependence of Magnetization}

The temperature dependence of magnetization (M-T) was measured on powdered samples using a VSM. Samples were slowly heated and cooled at a rate of approximately $10^{\circ} \mathrm{C} / \mathrm{min}$ in 
flowing $\mathrm{N}_{2}$ gas and measurements taken at $1^{\circ}$ intervals. Average values of $M$ were calculated by averaging the two magnetization values at each temperature along the heating and cooling curves. This was done for four separate M-T runs in fields of 498, 640, 748 , and $997 \mathrm{mT}$ and produced a series of M-H curves as a function of temperature. The comparable procedure of measuring M-H at different temperatures produced similar results but was much more time consuming.

Saturation magnetization (per gram) $M_{s}(T)$ was determined from the M-T,H data using three different methods: (1) $M$ versus $H^{-2}$, (2) $M$ versus $H$, and (3) $H / M$ versus $M^{2}$. The method used is important because each method predicts slightly different values of $M_{1}$ near $T_{c}$ and the results of the subsequent regression analysis are very sensitive in the region near $T_{c}$, where $M_{1}$ changes most rapidly.

Method 1. The first method of determining $M_{s}(T)$ fits straight lines to plots of $M$ versus $H^{-2}$ at constant temperature as suggested by the empirical approximation for the approach to saturation

$$
M(T)=M_{s}(T)\left(1-\frac{a}{H^{2}}\right)
$$

Here $M_{\mathrm{a}}$ is obtained as the intercept at $H^{-2}=0$ for each temperature [e.g., Cullity, 1972].

Method 2. The second method of determining $M_{s}(T)$ fits straight lines to plots of $M$ versus $H$ at constant temperature

$$
M(T)=M_{s}(T)+a H
$$

In this case, $M_{s}(T)$ is obtained as the intercept at $H=0$ for each temperature. According to McGuire and Flanders [1969], it is incorrect to use equation (A1) for temperatures above $T \delta$. The induced magnetization, which is proportional to $H$, becomes more significant than the $\mathrm{H}^{-2}$ term with increasing temperature. The condition $T S T / 2$ is satisfied for all the titanomagnetite compositions from room temperature and above and for magnetite above $150^{\circ} \mathrm{C}$. Differences in $M_{s}(T)$ between the two methods were negligible for pure magnetite, but became more noticeable for the Ti-rich titanomagnetites with lower Curie temperatures.

Method 3. The third method of determining $M_{d}(T)$ is described by Belov [1961] and McGuire and Flanders [1969] and is called the method of thermodynamic coefficients. This method is based on the Landau theory of second order phase transitions [e.g., Belov, 1961; Stanley, 1971]. It can also be derived from molecular field theory by a power series expansion of the Brillouin function near $T_{c}$ [Arrott, 1952]. Both derivations result in the equation

$$
H=\alpha(T)\left[M_{s}(T)+M_{i}(T)\right]+\beta(T)\left[M_{s}(T)+M_{i}(T)\right]^{3}
$$

where $\alpha(T)$ and $\beta(T)$ are the thermodynamic coefficients, $M_{i}$ is the induced magnetization, and $M=M_{\mathrm{z}}+M_{i}$ is the experimentally observed magnetization in field $H$. When $H=0, M_{i}=0$, and equation (A3) becomes

$$
M_{s}^{2}(T)=-\frac{\alpha(T)}{\beta(T)}
$$

For temperatures near $T_{c}$ curves of $H / M(T)$ versus $M(T)^{2}$ at various temperatures near $T_{c}$ should form a series of parallel straight lines, passing through the origin for $T=T_{c}$. A series of isothermal curves of $H / M$ versus $M^{2}$ is shown for TM60 in Figure A1. From these curves, $\alpha(T)$ is determined from the intercept on the $H / M$ axis and $\beta(T)$ is determined from the slope. The Curie temperature is then determined as the value of $T$ for which $\alpha=0$. A plot of $\alpha(T)$ is shown in the insert in Figure A1. Curie temperatures determined by this method for magnetite and titanomagnetites are given in Table A1.

Comparison of the three different methods of calculating $M_{s}(T)$ are shown in Figure A2 for TM60. As was the case for the other samples, methods 2 and 3 produce similar results at some distance away from the Curie temperature. However, as $T$ approaches $T_{c}$, the curves begin to diverge. The curve determined by method 3 drops sharply and goes to zero at $T_{c}$. The curves determined by the other two methods decrease with temperature more gradually near $T_{c}$ and have a high-temperature tail extending above $T_{c}$.

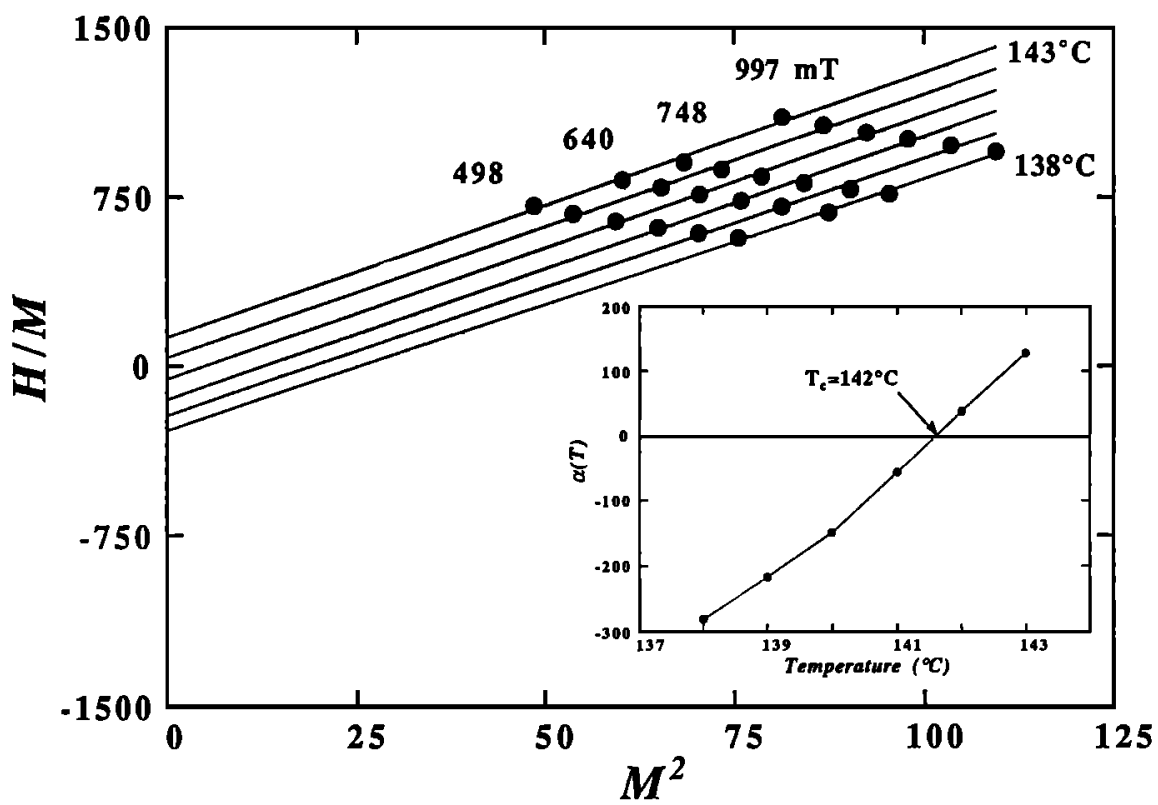

Fig. A1. An example of the method of thermodynamic coefficients for TM60 using equation (A3). The solid circles are the magnetization values for a particular value of field and temperature. The solid lines are the best linear fits from which the intercept $(\alpha(T))$ and slope $(\beta(T))$ can be determined. The insert is a plot of the intercepts $(\alpha(T))$ on the $H / M$ axis as a function of temperature. The Curie temperature is the temperature for which $\alpha=0$. 


\section{Curve Fitting Procedure}

Curie temperatures and power law exponents were also estimated from equations (9) and (10) in the text using nonlinear regression [Press et al., 1988] that included $T_{c}$ as an unknown parameter and temperature as the independent variable. However, the fit is sensitive to length of the temperature interval used. The temperature at which the fit is maximized is defined as the maximum cutoff temperature. Data above the cutoff temperature are not included in the fit. The effect of the cutoff temperature was noticeable for the $M_{\mathrm{a}}$ data because (1) the experimental values of $M$, do not go to zero at $T_{c}$ (except for method 3) but extend in a high temperature tail, due to measurement in a finite field, (see Figure A2), and (2) most of the variation in $M_{s}$ occurs near $T_{c}$. In contrast, $\lambda$, varies smoothly over the entire temperature interval and there is no high temperature tail. When fitting $\lambda_{r}$ data, the sensitivity to cutoff temperature was found to be negligible.

To obtain the best overall fit to equations (9) and (10), the strategy developed by Coe et al. [1978] for analyzing results from paleointensity experiments was used. The procedure was as follows. First, a series of nonlinear fits was calculated, each using a different cutoff temperature. The range of cutoff temperatures extended from $50^{\circ} \mathrm{C}$ below to $20^{\circ} \mathrm{C}$ above the Curie temperature estimated from the method of thermodynamic coefficients. The index of quality $Q$ was calculated as described by Coe et al. [1978]. This parameter takes into account (1) the fraction of the dependent variable $\left(M_{\mathrm{B}}\right.$ or $\left.\lambda_{\mathrm{n}}\right)$ spanned by the temperature interval defined by the cutoff temperature; (2) the spacing between data points; and (3) the relative uncertainty in the exponent. The fit with the highest value of $Q$ was then used as the best estimate for
$T_{c}$ and $n$. This procedure was applied to the magnetostriction data using equation (9) (Figures 9-12), the three different estimates of $M_{s}(T)$ using equation (10) (e.g., see Figure A2), and the linear least squares fitting of $\lambda_{2}$ and $M_{3}$ using equation (8). The results are summarized in Tables A1 to A3. Although the statistical

TABLE A1. Estimates of Curie Temperature Determined from Magnetostriction and Magnetization Data

\begin{tabular}{ccccc}
\hline Composition & \multicolumn{4}{c}{ Type of Data } \\
\cline { 2 - 5 } & $\lambda_{2}-T$ & $M-H$ & $M-H^{-2}$ & $M^{2}-H / M$ \\
\hline TM0A & 548 & 574 & 576 & 573 \\
TM0B & 567 & 574 & 576 & 573 \\
TM40 & 300 & 305 & 316 & 304 \\
TM60 & 149 & 141 & 148 & 142 \\
AMTM60 & 67 & 78 & 91 & 73 \\
\hline
\end{tabular}

TMOA and TMOB are the same sample but are treated separately for the magnetostriction results.

TABLE A2. Power Law Exponents for $M_{s} \propto\left(T_{c} T\right)^{n}$

\begin{tabular}{llll}
\hline Composition & \multicolumn{3}{c}{ Type of Data } \\
\cline { 2 - 4 } & $M-H$ & $M-H^{-2}$ & $M^{2}-H / M$ \\
\hline TM0 & $0.39(1)$ & $0.36(1)$ & $0.38(1)$ \\
TM40 & $0.35(2)$ & $0.35(3)$ & $0.37(1)$ \\
TM60 & $0.44(4)$ & $0.42(6)$ & $0.47(2)$ \\
AMTM60 & $0.53(10)$ & $0.56(15)$ & $0.46(6)$ \\
\hline
\end{tabular}

Numbers in parentheses refer to standard deviation in least significant units.

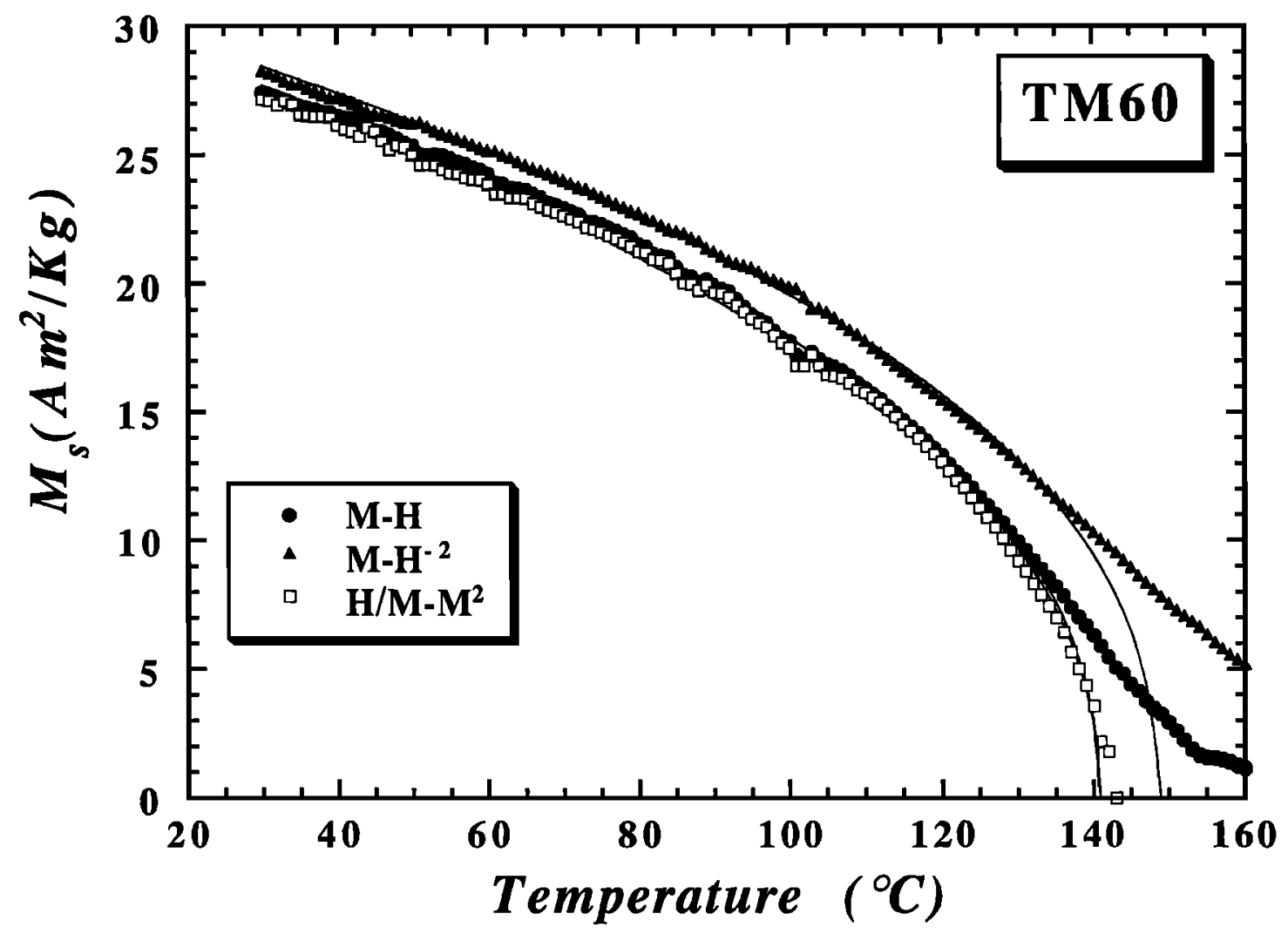

Fig. A2. Comparison of three methods of calculating saturation magnetization $\left(M_{2}\right)$ from $M-H, T$ data for TM60. Solid triangles are data derived using method $1\left(M-H^{-2}\right)$, solid circles are data derived using method $2(M-H)$, and open squares are data derived using method $3\left(M^{2}-H / M\right)$. Solid lines are power law fits to the equation $(1-T / T)^{n}$. 
TABLE A3. Power Law Exponents for the Thermal Dependence of Magnetostriction as a Function of Magnetization

\begin{tabular}{|c|c|c|c|c|c|c|}
\hline \multirow[t]{2}{*}{ Composition } & \multicolumn{2}{|c|}{$\mathbf{M}-\mathbf{H}$} & \multicolumn{2}{|c|}{$\mathbf{M}-\mathrm{H}^{-2}$} & \multicolumn{2}{|c|}{$M^{2}-H / M$} \\
\hline & $n_{3} / n_{2}$ & $n_{1}$ & $n_{y} / n_{2}$ & $n_{1}$ & $n_{3} / n_{2}$ & $n_{1}$ \\
\hline TMOA & $2.31(6)$ & $2.69(1)$ & $2.50(8)$ & $2.81(1)$ & $2.37(6)$ & $2.66(1)$ \\
\hline TMOB & $2.21(6)$ & $2.25(1)$ & $2.39(7)$ & $2.46(1)$ & $2.26(9)$ & $2.23(1)$ \\
\hline TM40 & $3.69(25)$ & $3.88(2)$ & $3.76(56)$ & $4.22(1)$ & $3.49(10)$ & $3.78(2)$ \\
\hline TM60 & $2.91(27)$ & $2.28(3)$ & $3.05(44)$ & $3.02(3)$ & $2.72(12)$ & $2.51(4)$ \\
\hline AMTM60 & $2.43(46)$ & $3.65(6)$ & $2.30(61)$ & $6.66(21)$ & $2.80(34)$ & $3.86(7)$ \\
\hline
\end{tabular}

Exponents $n_{1} / n_{2}$ determined by equations (10)-(12) and $n_{1}$ determined by equation (9) in text. Numbers in parentheses refer to standard deviation in least significant units.

robustness of this procedure may be questionable, it did provide a useful and objective approach to the problem.

There is good agreement among the various methods for calculating both $T_{c}$ and $n$ as shown in Tables A1 to A3. The agreement is poorest for AMTM60, probably because its Curie temperature is so near room temperature. Here, all the experimental data were measured within the temperature interval in which $M_{s}(T)$ is changing most rapidly and, therefore, is most sensitive to choice of method. The other "anomalous" value is the low apparent Curie temperature calculated from the magnetite magnetostriction data for the TM0A runs. However, the maximum temperature of measurement was just $480^{\circ} \mathrm{C}$, well below the known Curie temperature of $575^{\circ} \mathrm{C}$. The curve fitting may not be reliable enough to extrapolate the remaining $100^{\circ}$, or the data themselves may not be reliable at the highest temperatures due .o short circuiting of the strain gage. This latter problem may produce a faster decrease in apparent magnetostriction than expected.

The numerical calculations also show that the $M-H^{-2}$ method predicts the highest values of $T_{c}$ (see Table A1) because $M_{s}(T)$ determined by this method produces the largest high-temperature tail above $T_{\epsilon}$. By contrast, the magnetostriction data estimates the lowest values of $T_{c}$, except for TM60. The asymptotic shape and low values of $\lambda_{s}(T)$ as $T$ approaches $T_{c}$ probably account for the lower estimates of $T_{c}$ determined by the curve-fitting procedures.

The power law exponents for the dependence of magnetostriction on magnetization are summarized in Table A3. Most of the variation in the exponents is attributable to the particular method for determining $M_{s}(T)$. In addition, the scatter in the values of the exponents determined by directly fitting $M_{\mathrm{a}}$ and $\lambda_{\mathrm{z}}$ (equation (8) in text and columns $n_{1}$ in Table A3) for the same sample are correlated with the difference between $T_{c}$ estimated from the $\lambda_{\mathrm{q}}$ and $M_{\mathrm{s}}$ data (Table A1). This trend suggests that the larger the difference between $T_{c}$ estimates, the higher the exponent. The double exponent method (equations (10) to (12) in text and columns $n_{3} / n_{2}$ in Table A3) effectively corrects for the difference in $T_{c}$ values and, hence, the scatter between methods is reduced.

Acknowledgments. Several people contributed time, expertise, and equipment to this project, most of which was performed while I was at the University of California,Davis. First, and foremost, I am grateful to Dyke Andreasen (UCD) for his patience and tenacity dealing with very small strain gages and a finicky magnetostrictometer. I am indebted to E.M. Gyorgy (AT\&T Bell Laboratories) for the long-term loan of the magnetostrictometer, to Brian Bonner (Lawrence Livermore National Laboratory) for use of his lock-in amplifier, to Robert Sheldon (Physics Department, UCD) for use of various electronic equipment, and to Ken Verosub (UCD) for help and support and the use of his laboratory. I also wish to thank Charles Lawson (USGS) for synthesizing the polycrystalline samples, Morris Young (LLNL) for growing the magnetite single- crystal, Gordon
Nord (USGS) for orienting the single- crystal, and Peter Schiffman (UCD) for the electron microprobe analysis. Finally, I thank B.J. Wanamaker, Ron Merrill, Subir Banerjee, and Phil Flanders for helpful discussions and suggestions. This research was supported by National Science Foundation grants EAR-8803622 and EAR-9017389. This is contribution no. 9202 of the Institute for Rock Magnetism. Support for the IRM is provided by grants from the Keck Foundation and the National Science Foundation.

\section{REFERENCES}

Appel, E., Stress anisotropy in Ti-rich titanomagnetites, Phys. Earth Planet. Inter., 46, 233-240, 1987.

Appel, E., and H. C. Soffel, Model for the domain state of Ti-rich titanomagnetites, Geophys. Res. Lett., 11, 189-192, 1984.

Arroth, A., Criterion of ferromagnetism from observations of magnetic isotherms, Phys. Rev., 108, 1394, 1952.

Belov, K.P., Magnetic Transitions, 154 pp., Consultants Bureau, New York, 1959.

Bickford, L., J. Pappis, and J. Stull, Magnetostriction and permeability of magnetite and cobalt-substituted magnetite, Phys. Rev., 99, 1210-1214, 1955.

Callen, E., and H. Callen, Static magnetoelastic coupling in cubic crystals, Phys. Rev., 129, 578-593, 1963.

Coe, R.S., S. Grommé, and E. A. Mankinen, Geomagnetic paleointensities from radiocarbon-data lava flows on Hawaii and the question of the Pacific nondipole low, J. Geophys. Res., 83, 1740-1756, 1978.

Collins, M.F., and D.H. Saunderson, Spin wave and critical fluctuations in magnetite, J. Appl. Phys., 41, 1433-1434, 1970.

Cullity, B. D., Introduction to Magnetic Materials, 666 pp., AddisonWesley, Reading, Mass., 1972.

Flanders, P., Magnetostriction in some magnetic oxide compacted powders, IEEE Trans. Magn., 10, 1050-1052, 1974.

Fletcher, E., and W. O'Reilly, Contribution of $\mathrm{Fe}^{2+}$ ions to the magnetocrystalline anisotropy constant $\mathrm{K}_{1}$ of $\mathrm{Fe}_{3-\mathrm{T}} \mathrm{Ti}_{\mathbf{x}} \mathrm{O}_{4}(\mathrm{O}<\mathrm{x}<0.1)$, J. Phys. C Solid State Phys., 7, 171-178, 1974.

Halgedahl, S. L., Domain pattern observations in rock magnetism: Progress and problems, Phys. Earth Planet. Inter., 46, 127-163, 1987.

Halgedahl, S. L., Magnetic domain patterns observed on synthetic Ti-rich titanomagnetite as a function of temperature and in states of thermoremanent magnetization, J. Geophys. Res., 96, 3943-3972, 1991.

Heider, F., and W. Williams, Note on temperature dependence of exchange constant in magnetite, Geophys. Res. Lett, 15, 184-187, 1988.

Hodych, J. P., Magnetostrictive control of coercive force in multidomain magnetite, Nature, 298, 542-544, 1982.

Hodych, J. P., Evidence for magnetostrictive control of intrinsic susceptibility and coercive force of multidomain magnetite in rocks, Phys. Earth Planet. Inter., 42, 184-194, 1986.

Hoekstra, B., E. Gyorgy, and G. Zydzik, Magnetostriction measurements with a recording rotating field magnetostrictometer, Rev. Sci. Instrum., 48, 1253-1255, 1977. 
Klapel, G., and P. Shive, High-temperature magnetostriction of magnetite, J. Geophys. Res., 79, 2629-2633, 1974.

Klerk, J., V. A. M. Brabers, and A. J. M. Kuipers, Magnetostriction of the mixed series $\mathrm{Fe}_{3-2} \mathrm{Ti}_{x} \mathrm{O}_{4}$ J. Phys. C Solid State Physics, 38, 187-189, 1977.

McGuire, T.R., and P.J. Flanders, Direct current magnetic measurements, in Magnetism and Metallurgy, vol. 1, edited by A.E. Berkowitz and E. Keller, pp. 124-189, Academic Press, New York, 1969.

Moon, T. S., Domain states in fine particle magnetite and titanomagnetite, J. Geophys. Res., 96, 9909-9923, 1991.

Moskowitz, B. M., and S. L. Halgedahl, Theoretical temperature and grain-size dependence of domain state in $x=0.6$ titanomagnetite, $J$. Geophys. Res., 92, 10,667-10,682, 1987.

Moskowitz, B. M., S. L. Halgedahl, and C. A. Lawson, Magnetic domains on unpolished and polished surfaces of titanium-rich titanomagnetite, $J$. Geophys. Res., 93, 3372-3386, 1988.

O'Reilly, W., Rock and Mineral Magnetism, 230 pp., Blackie, Glasgow, 1984.

Press, W.H., B.P. Flannery, S.A. Teukolsky, and W.T. Vetterling, Numerical Recipes in C: The Art of Scientific Computing, 735 pp., Cambridge University Press, New York, 1988.
Stacey, F., and K. Wise, Crystal dislocations and coercivity in fine grained magnetite, Aust. J. Phys., 20, 507-513, 1967.

Stanley, H.E., Introduction to Phase Transitions and Critical Phenomene, 308 pp., Oxford University Press, New York, 1971.

Syono, $\mathrm{Y}$., Magnetocrystalline anisotropy and magnetostriction of $\mathrm{Fe}_{3} \mathrm{O}_{4}$ $-\mathrm{Fe}_{2} \mathrm{TiO}_{4}$ series, with special application to rock magnetism, Jpn. $J$. Geophys., 4, 71-143, 1965.

Xu, S., and R. T. Merrill, Microstress and microcoercivity in multidomain grains, J. Geophys. Res., 94, 10,627-10,636, 1989.

$\mathrm{Xu}, \mathrm{S}$., and R. T. Merrill, Microcoercivity, bulk coercivity, and saturation remanence in multidomain materials, J. Geophys. Res., 95, 7083-7090, 1990.

B.M. Moskowitz, Department of Geology and Geophysics, University of Minnesota at Minneapolis-St. Paul, Minneapolis, MN 55455.

(Received February 20, 1992; revised July 27,1992 ;

accepted August 3, 1992.) 